\title{
Selection of internal reference genes for SYBR green qRT-PCR studies of rhesus monkey (Macaca mulatta) tissues Kung Ahn ${ }^{\dagger 1}$, Jae-Won Huh ${ }^{\dagger 1,2}$, Sang-Je Park ${ }^{\dagger 1}$, Dae-Soo Kim³ ${ }^{3}$, Hong- Seok Ha1, Yun-Ji Kim¹, Ja-Rang Lee ${ }^{1}$, Kyu-Tae Chang ${ }^{2}$ and Heui-Soo Kim*1,3
}

Address: ${ }^{1}$ Division of Biological Sciences, College of Natural Sciences, Pusan National University, Busan, 609-735, Republic of Korea, ${ }^{2}$ National Primate Research Center (NPRC), KRIBB, Ochang, Chungbuk, 363-883, Republic of Korea and ${ }^{3}$ PBBRC, Interdisciplinary Research Program of Bioinformatics, College of Natural Sciences, Pusan National University, Busan, 609-735, Republic of Korea

Email: Kung Ahn - ankung79@pusan.ac.kr; Jae-Won Huh - ativan76@kribb.re.kr; Sang-Je Park - sangje82@pusan.ac.kr; DaeSoo Kim - kds2465@pusan.ac.kr; Hong-Seok Ha - hay2k@pusan.ac.kr; Yun-Ji Kim - ky2j1020@pusan.ac.kr; Ja-Rang Lee - jr1006@pusan.ac.kr; Kyu-Tae Chang - changkt@kribb.re.kr; Heui-Soo Kim* - khs307@pusan.ac.kr

* Corresponding author †Equal contributors

Published: 10 September 2008

BMC Molecular Biology 2008, 9:78 doi:10.1 I86//47|-2199-9-78
Received: 18 March 2008

Accepted: 10 September 2008

This article is available from: http://www.biomedcentral.com/I47/-2/99/9/78

(C) 2008 Ahn et al; licensee BioMed Central Ltd.

This is an Open Access article distributed under the terms of the Creative Commons Attribution License (http://creativecommons.org/licenses/by/2.0), which permits unrestricted use, distribution, and reproduction in any medium, provided the original work is properly cited.

\begin{abstract}
Background: The rhesus monkey (Macaca mulatta) is a valuable and widely used model animal for biomedical research. However, quantitative analyses of rhesus gene expression profiles under diverse experimental conditions are limited by a shortage of suitable internal controls for the normalization of mRNA levels. In this study, we used a systematic approach for the selection of potential reference genes in the rhesus monkey and compared their suitability to that of the corresponding genes in humans.
\end{abstract}

Results: Eight housekeeping genes (HKGs) (GAPDH, SDHA, ACTB, RPLI3A, RPL32, UBA52, PGKIY, and $Y W H A Z$ ) from rhesus monkeys and humans were selected to test for normalization of expression levels in six different tissue types (brain, colon, kidney, liver, lung, and stomach). Their stability and suitability as reference genes were validated by geNorm, NormFinder and BestKeeper programs. Intriguingly, RPLI $3 A$ and RPL 32 were selected as ideal reference genes only in rhesus monkeys.

Conclusion: The results clearly indicated the necessity of using different reference genes for normalization of expression levels between rhesus monkeys and humans in various tissues.

\section{Background}

The rhesus monkey (Macaca mulatta) has been one of the most valuable experimental model species for biomedical studies in various research fields including microbiology, neuroscience, and biochemistry. Recently, the genome sequence of the rhesus monkey was determined by the Rhesus Macaque Genome Sequencing and Analysis Consortium [1]. The consortium revealed that the average sequence similarity between humans and rhesus monkeys is $93 \%$. Approximately twenty thousand genes were annotated or predicted by the human-based comparative analysis. In order to conduct biomedical research in areas such as infectious disease, cancer research, and drug development using various monkey tissues, quantitative gene expression analysis is essential.

Quantitative real-time PCR (qRT-PCR) is a recently developed and popularized molecular technique that can be 
used for gene expression studies that require highly sensitive and accurate quantification of mRNA levels from various samples [2]. These PCR-based analyses allow investigators to analyze a limited amount of material of interest while guaranteeing accurate quantification of target mRNAs. However, to achieve optimal results in qRTPCR analysis, certain steps are indispensable including: (1) quality control of the material of interest and the primer sets, (2) determination of the PCR efficiency, and (3) selection of suitable internal control genes for normalization [3]. The availability of advanced biomaterials including sophisticated PCR instrumentation, kits for RNA isolation from various materials, and highly purified Taq polymerase, has overcome many of these difficult steps with the exception of identifying appropriate internal control genes [4]. Different expression profiling results from the same target genes can be obtained by using different HKGs as internal references [5]. Therefore, selection of proper HKGs is important for the accurate analysis and normalization of data generated by real-time qRT-PCR [2].

Before the systematic selection of reference genes, researchers interested in quantitative expression analysis have used empirically determined housekeeping genes such as ACTB and GAPDH. However, recent studies have provided solid evidence that transcription levels of housekeeping genes vary between cell types [6-11], developmental stages $[12,13]$, and experimental conditions $[14,15]$, individuals $[16-20]$. Therefore, stable reference gene selection procedures have to be undertaken before quantitative mRNA expression analysis can be conducted.

In this study, three different specific tools (geNorm, Normfinder, and BestKeeper) that can be used for the validation of the stability of selected HKGs (GAPDH, SDHA, ACTB, RPL13A, RPL32, UBA52, PGK1Y, and YWHAZ) were investigated in rhesus monkey and human tissues (brain, colon, kidney, liver, lung, stomach) using realtime qRT-PCR with SYBR green.

\section{Results and discussion \\ Reference gene and qRT-PCR primer selection}

For the selection of suitable reference genes from the human and rhesus monkey genomes, the eight most commonly used and available HKGs were selected: Hydroxymethylbilane synthase (HMBS), Beta-actin (ACTB), Glyceraldehyde-3-phosphate dehydrogenase (GAPDH), Ribosomal protein 13 (RPL13A), Ribosomal protein 32 (RPL32), Tyrosine 3-monooxygenase (YWHAZ), Hypoxanthine phosphoribosyltransferase 1(HPRT1), and TATA box-binding protein (TBP). Rhesus monkey HKGs are identified by comparison of orthologous loci of the human HKGs. Summarized sequence information for the human and rhesus monkey genes is shown in Table 1. This gene information was also used to design qRT-PCR primers (Table 2).

\section{Amplification efficiency}

The expression levels of HKGs were analyzed using the relative quantification (delta-Ct method) [21]. The amplification efficiencies and correlation coefficients $\left(\mathrm{R}^{2}\right)$ of the eight HKGs were generated using the slopes of the standard curves obtained by serial dilutions. Standard curves with a ten-fold dilution series were used to calculate the amplification efficiency (additional files 1 and 2). Similar amplification efficiencies for the corresponding human and rhesus monkey reference genes are a prerequisite for accurate real-time RT-PCR quantification. The amplification efficiency was calculated by the formula: efficiency $(\%)=\left(10^{(-1 / \text { slope })}-1\right) * 100$. The efficiency range of the qRTPCR amplifications for all of the genes tested was between $80 \%$ and $115 \%$. Overall, similar efficiency levels were observed between human and rhesus samples (additional

Table I: GenBank accession numbers and functions of 8 reference genes in rhesus monkeys and humans

\begin{tabular}{|c|c|c|c|c|}
\hline \multirow[t]{2}{*}{ Symbol } & \multirow[t]{2}{*}{ Gene Name } & \multirow[t]{2}{*}{ Function } & \multicolumn{2}{|c|}{ Accession Number } \\
\hline & & & Human & Rhesus \\
\hline GAPDH & Glyceraldehyde-3-phospate dehydrogenase & Glycolitic enzyme & NM 002046.3 & $\underline{X M 001105471.1}$ \\
\hline ACTB & Beta-actin & Cytoskeletal structural protein & NM 001101.2 & NM 001033084.1 \\
\hline RPLI3A & Ribosomal protein LI3A & Member of ribosome protein & NM 012423.2 & $\underline{X M 001093017.1}$ \\
\hline RHL32 & 60 S ribosomal protein $\mathrm{L} 32$ & Member of ribosome protein & NM 001007074.1 & XM 001117016.1 \\
\hline YWHAZ & Tyrosine 3-monooxygenase & $\begin{array}{l}\text { Signal transduction by binding to phosphorilated } \\
\text { serine residue on a variaty of signaling molecules }\end{array}$ & NM 145690.I & XM 001098275.1 \\
\hline HMBS & hydroxymethylbilane synthase & $\begin{array}{l}\text { Third enzyme of the heme biosynthetic pathway } \\
\text { and catalyzes the head to tail condensation of } \\
\text { four porphobilinogen molecules into the linear } \\
\text { hydroxymethylbilane. }\end{array}$ & NM 000190.3 & $\underline{X M 001101850.1}$ \\
\hline TBP & TATA box binding protein & $\begin{array}{l}\text { Transcription initiation from RNA polymerase II } \\
\text { promoter }\end{array}$ & NM 003194.3 & $\underline{X M 001085016.1}$ \\
\hline HPRTI & Hypoxanthine phosphoribosyltransferase I & Metabolic salvage of purines in mammals & NM 000194.2 & XM 001097691.1 \\
\hline
\end{tabular}


Table 2: Details of primers used for quantitative RT-PCR

\begin{tabular}{|c|c|c|c|c|c|}
\hline Gene & Forward Primer Sequence [ $\left.5^{\prime} \rightarrow 3^{\prime}\right]$ & Position in cDNA & Reverse Primer Sequence [5' $\rightarrow$ 3'] & Position in cDNA & Products Size \\
\hline GAPDH & GAAATCCCATCACCATCTTCCAGG & 4th Exon & GAGCCCCAGCCTTCTCCATG & 5th Exon & $120 \mathrm{bp}$ \\
\hline ACTB & CCTGGCACCCAGCACAAT & 4th Exon & GGGCCGGACTCGTCATAC & 5th Exon & 144 bp \\
\hline RPLI3A & CCTGGAGGAGAAGAGGAAAGAGA & 7th Exon & TTGAGGACCTCTGTGTATTTGTCAA & 8th Exon & 126 bp \\
\hline RPL32 & CAACATTGGTTATGGAAGCAACA & 3th Exon & TGACGTTGTGGACCAGGAACT & 3th Exon & $80 \mathrm{bp}$ \\
\hline YWHAZ & TCCTTTGCTTGCATCCCAC & 6th Exon & AAGGCAGACAATGACAGACCA & 6th Exon & $132 \mathrm{bp}$ \\
\hline HMBS & ACCAAGGAGCTTGAACATGC & 5th Exon & GAAAGACAACAGCATCATGAG & 7th Exon & 145 bp \\
\hline TBP & TGCACAGGAGCCAAGAGTGAA & 5,6 th Exon & CACATCACAGCTCCCCACCA & 6th Exon & 132 bp \\
\hline HPRTI & AATTATGGACAGGACTGAACGTCTTGCT & 2, 3th Exon & TCCAGCAGGTCAGCAAAGAATTTATAGC & 3th Exon & 117 bp \\
\hline
\end{tabular}

file 3). The amplification specificity for each qRT-PCR analysis was confirmed by melting curve analysis (additional files 4 and 5).

\section{Data analysis}

We analyzed eight housekeeping genes for the selection of stable HKGs using three authorized programs (geNORM, NormFinder, and BestKeeper) [22-24].

\section{geNorm}

The normalization step must be conducted very carefully for accurate gene expression level analysis. However, the selection of stable HKGs for normalization is very complicated. geNorm is a useful tool for the selection of the most stable internal control genes using the principle that the expression ratio of two ideal internal control genes is identical in all tested samples [22].

Using the geNorm program, the eight HKGs selected for normalization were ranked according to their $M$ values. The $M$ value is the average pair-wise variation of a particular gene with all other control genes. The $M$ values for RPL13A, RPL32, TBP, HMBS, ACTB, YWHAZ, HPRT1, and GAPDH in rhesus were lower than 1.5.RPL32 and RPL13A displayed the most stability of all eight genes according to geNorm analysis. Both genes encode components of the $60 \mathrm{~S}$ ribosomal subunit that is involved in protein biosynthesis (Fig. 1a). In contrast to rhesus monkeys, human tissue analysis indicated that GAPDH and HMBS were the most stable genes (Fig. 1c). The $M$ values of all tested human genes except for HPRT1 were lower than 1.5. To investigate the optimal number of reference genes, it was determined whether the stepwise inclusion of less stable genes significantly affected the normalization factors, considering the term $\mathrm{V}$ (Fig. 1).

As the most stable reference genes, RPL32 and RPL13A yielded a V value of 0.200 in the rhesus monkey (Fig. 1b). That value indicated that it would not be necessary to include additional reference genes in qRT-PCR of rhesus monkey tissues. However, in the case of humans, the addition of a third and fourth gene significantly affected the reliability of the selected HKGs (Fig. 1d).
The results of the geNorm tool indicated different results between humans and rhesus monkeys. RPL32 and RPL13A in the rhesus monkey and GAPDH, HMBS, ACTB and RPL32 in humans are the most stable HKGs for the accurate calculation of normalization factors.

\section{NormFinder}

NormFinder is another reference tool for identifying the ideal normalization genes among various candidate HKGs [23]. NormFinder ranks the various candidate reference genes according to their expression variation between inter and intra groups. This analysis identified HMBS and RPL32 as the most stable genes in rhesus monkeys and humans, respectively (Table 3 ). These rankings appear to be generally different from the results of geNorm. However, RPL32, GAPDH and ACTB in humans and HMBS and RPL32 in rhesus monkeys are still ranked as the most stable reference genes. Although geNorm and NormFinder did not identify the exact same reference genes, GAPDH in humans and HMBS in rhesus monkeys are still considered candidate reference genes.

Intriguingly, human HMBS showed opposite results between geNorm and Normfinder program. In the geNorm software, the most important factor is gene expression stability (M). The principle of geNorm is that the expression ratio of two ideal internal control genes is identical in all tested samples [22]. Thus, the geNorm program did not consider the other HKGs. However, Normfinder program focuses on the two genes with the least intra-and intergroup expression variation. In other words, the Normfinder program considered other HKGs for the selection of good HKGs. For example, high rate of expression variation of HMBS among HKGs could result in the discordance between Normfinder and geNorm (Fig. 2).

\section{BestKeeper}

The BestKeeper program index was created using the geometric mean of each candidate gene's Ct values [24]. Initial analysis of the data, based on the inspection of raw $\mathrm{Ct}$ values, calculated variations for all the housekeeping genes in the six samples (SD $[ \pm \mathrm{Ct}]$ and $\mathrm{CV}[\% \mathrm{Ct}])$, and showed an overall stability in gene expression. Genes with 


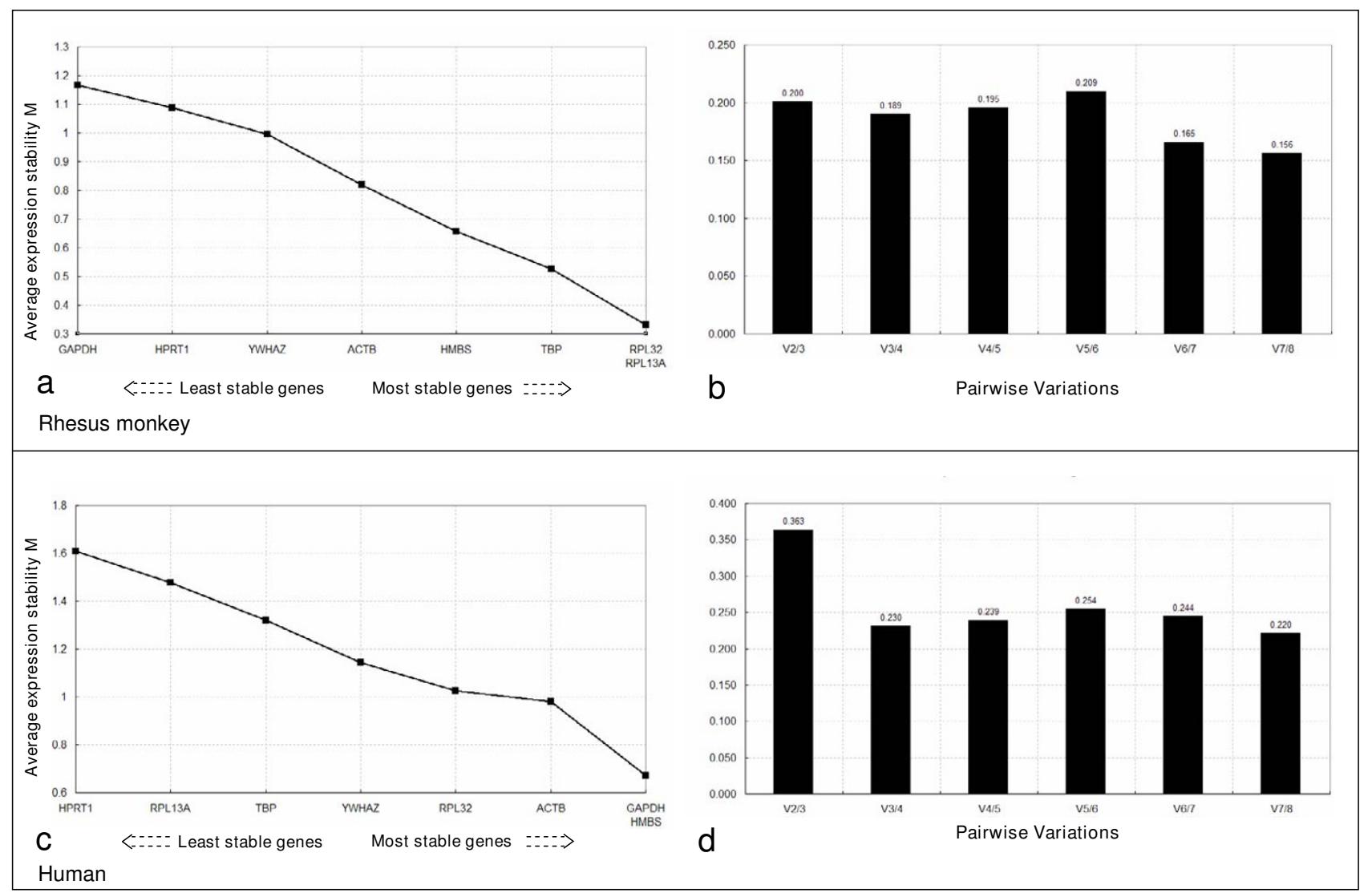

\section{Figure I}

Selection of the most suitable reference genes for normalization in rhesus monkey (a and b) and human (c and d) samples using geNorm analysis. Stepwise exclusion of the least stable genes by calculating the average expression stability ( $a$ and $c$ ) was conducted. The value of $M$ was calculated for each gene, and the least stable gene with the highest $M$ value was automatically excluded for the next round of calculations. The $x$-axis from left to right indicates the ranking of the genes according to their expression stability. Determination of the optimal number of reference genes for normalization (b and $d$ ) was conducted. The software calculates the normalization factor from at least two genes at which the variable $V$ defines the pair-wise variation between two sequential normalization factors.

SD values higher than 1 were considered inconsistent. ACTB, HMBS, RPL13A, RPL32, TBP, YWHAZ showed a $\mathrm{SD}$ value lower than 1 in the rhesus monkey. Among them, RPL13A (SD = 0.22) had the lowest Ct value variation. GAPDH had the highest $\mathrm{Ct}$ value variation. In human, HMBS and RPL32 exhibit SD values lower than 1.

Table 3: Candidate reference genes for normalization and the best combination of two genes listed according to their expression stability calculated by the NormFinder program

\begin{tabular}{ccccc}
\hline Ranking order & Gene name & Stablility value of rhesus monkey & Gene name & Stablility value of human \\
\hline 1 & HMBS & 0.349 & RPL32 & 0.39 \\
2 & RPL32 & 0.4 & GAPDH & 0.594 \\
3 & RPLI3A & 0.434 & ACTB & 0.668 \\
4 & ACTB & 0.589 & TBP & 0.705 \\
5 & HPRTI & 0.649 & YWHAZ & 0.754 \\
6 & TBP & 0.687 & HMBS & 0.936 \\
7 & YWHAZ & 0.709 & RPLI3A & 1.073 \\
8 & GAPDH & 0.837 & HPRTI & 1.185
\end{tabular}




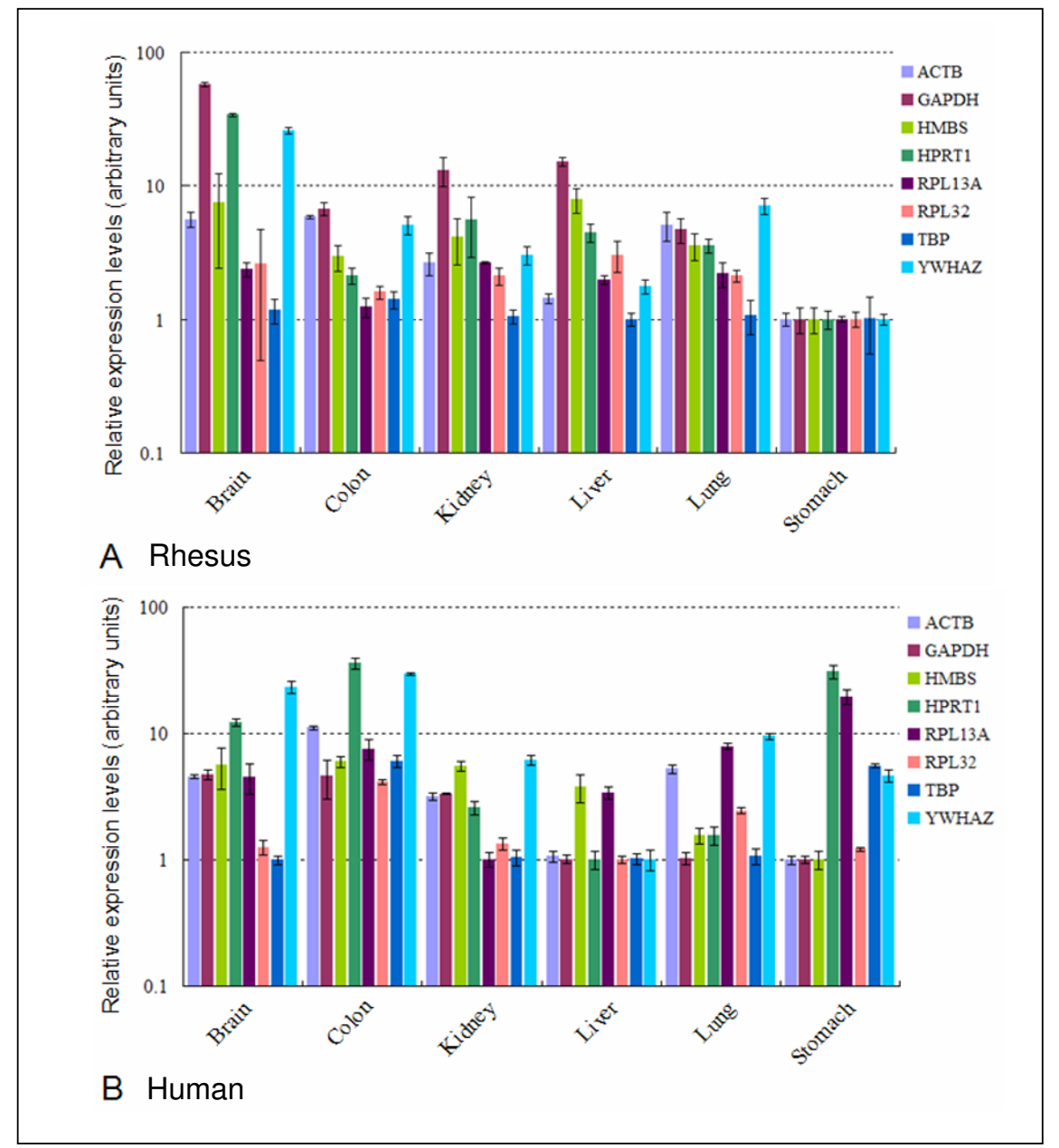

Figure 2

Logarithmic histogram of the expression levels of 8 internal reference genes determined in 6 different tissues. Relative expression levels of rhesus monkey $(A)$ and human $(B)$ with six different tissues. The respective bar and genes name are: actin beta (ACTB: light blue bar), Glyceraldehyde-3-phospate dehydrogenase (GAPDH: light purple bar), Hydroxymethylbilane synthase (HMBS: light green bar), Hypoxanthine phosphoribosyltransferas I (HPRTI: green bar) Ribosomal protein LI3A (RPLI3A: dark purple bar), 60S ribosomal protein L32 (RPL32: pink bar), TATA box binding protein (TBP: dark blue bar), Tyrosine 3-monooxygenase (YWHAZ: sky blue bar).

RPL32 had the lowest Ct value variation and HPRT1 had the highest $\mathrm{Ct}$ value variation (Table 4). In the case of HMBS and RPL32, BestKeeper also showed a different result compared to Normfinder and geNorm program, respectively. Because the variation of $\mathrm{Ct}$ value of $H M B S$ and RPL32 is ranked very low, the BestKeeper program indicated HMBS and RPL32 as good candidate HKGs. However, compared to BestKeeper, geNorm is considered a pair-wise variation between the gens as the most important factor. Thus geNorm did not indicate one gene as a best reference gene but indicated a gene pair.
Based on the Bestkeeper program, the RPL13A gene in the rhesus monkey and the RPL32 gene in the human were selected as the best reference genes.

\section{Validation}

To validate our results from the rhesus monkey, one unknown rhesus monkey was tested using same reference genes. And results were analyzed for the selection of stable HKGs using three authorized programs (geNORM, NormFinder, and BestKeeper) (additional files 6, 7 and 8). Although, slight differences were observed, overall patterns were coincided with our results of RPL13A and RPL32 as ideal reference genes in rhesus monkeys. 
Table 4: Results from Bestkeeper descriptive statistical analysis

\begin{tabular}{|c|c|c|c|c|c|c|c|c|c|c|c|c|c|c|c|c|}
\hline & \multicolumn{2}{|c|}{ GAPDH } & \multicolumn{2}{|c|}{ ACTB } & \multicolumn{2}{|c|}{ RPLI3A } & \multicolumn{2}{|c|}{ RPL32 } & \multicolumn{2}{|c|}{ YWHAZ } & \multicolumn{2}{|c|}{ HMBS } & \multicolumn{2}{|c|}{ TBP } & \multicolumn{2}{|c|}{ HPRTI } \\
\hline \multirow[t]{2}{*}{$\mathrm{n}$} & \multicolumn{2}{|c|}{6} & \multicolumn{2}{|c|}{6} & \multicolumn{2}{|c|}{6} & \multicolumn{2}{|c|}{6} & \multicolumn{2}{|c|}{6} & \multicolumn{2}{|c|}{6} & \multicolumn{2}{|c|}{6} & \multicolumn{2}{|c|}{6} \\
\hline & human & $\begin{array}{l}\text { rhesus } \\
\text { monkey }\end{array}$ & human & $\begin{array}{l}\text { rhesus } \\
\text { monkey }\end{array}$ & human & $\begin{array}{l}\text { rhesus } \\
\text { monkey }\end{array}$ & human & $\begin{array}{l}\text { rhesus } \\
\text { monkey }\end{array}$ & human & $\begin{array}{l}\text { rhesus } \\
\text { monkey }\end{array}$ & human & $\begin{array}{l}\text { rhesus } \\
\text { monkey }\end{array}$ & human & $\begin{array}{l}\text { rhesus } \\
\text { monkey }\end{array}$ & human & $\begin{array}{l}\text { rhesus } \\
\text { monkey }\end{array}$ \\
\hline $\begin{array}{r}\text { geo Mean } \\
{[\mathrm{CP}]}\end{array}$ & 17.01 & 15.79 & 15.03 & 16.31 & 19.31 & 16.83 & 22.43 & 25.36 & 19.30 & 20.7 I & 27.06 & 29.87 & 19.65 & 17.53 & 22.89 & 23.47 \\
\hline $\begin{array}{r}\text { ar Mean } \\
\text { [CP] }\end{array}$ & 17.04 & 15.86 & 15.08 & 16.31 & 19.36 & 16.83 & 22.44 & 25.37 & 19.34 & 20.72 & 27.08 & 29.88 & 19.71 & 17.54 & 22.98 & 23.50 \\
\hline $\min [C P]$ & 15.84 & 13.97 & 13.25 & 15.75 & 17.43 & 16.53 & 21.12 & 24.44 & 17.62 & 19.87 & 26.19 & 28.26 & 17.74 & 17.10 & 20.44 & 21.87 \\
\hline $\max [\mathrm{CP}]$ & 18.08 & 17.85 & 16.65 & 16.92 & 21.71 & 17.28 & 23.16 & 25.81 & 20.22 & 21.42 & 28.76 & 30.95 & 22.63 & 18.15 & 25.61 & 24.71 \\
\hline $\begin{array}{r}\text { std dev }[ \pm \\
\mathrm{CP}]\end{array}$ & 1.02 & I.34 & 1.04 & 0.30 & 1.04 & 0.22 & 0.63 & 0.35 & 1.10 & 0.54 & 0.91 & 0.72 & 1.31 & 0.30 & 1.95 & 1.04 \\
\hline $\begin{array}{r}\mathrm{CV}[\% \\
\mathrm{CP}]\end{array}$ & 6.00 & 8.45 & 6.92 & 1.85 & 5.37 & 1.33 & 2.79 & 1.39 & 5.71 & 2.60 & 3.35 & 2.41 & 6.62 & 1.73 & 8.49 & 4.41 \\
\hline
\end{tabular}

\section{Conclusion}

In this study, we investigated for the first time the most reliable HKGs for the normalization of real-time qRT-PCR data in rhesus monkey tissues (brain, colon, kidney, liver, lung, stomach) using three programs (geNorm, Normfinder, and Bestkeeper). The results from the rhesus monkey were compared to those of humans.

Our results indicated that the two most stable genes, RPL13A and RPL32, covering a broad expression range, could be used as reference genes for relative gene quantification and normalization purposes in gene profiling studies of the rhesus monkey as determined by three reference gene identification programs.

In humans, RPL32, HMBS and GAPDH were found to be suitable reference genes. In the case of RPL32, two programs (Bestkeeper and Normfinder) identified this gene as a reliable reference gene. However, geNorm indicated that RPL32 was an unsuitable reference gene by virtue of having a high $\mathrm{M}$ value. Likewise, the GAPDH gene was rejected by the Bestkeeper program as a result of having a high SD $[ \pm \mathrm{Ct}]$ value. Finally, HMBS was ranked as the sixth best gene by the Normfinder program.

Intriguingly, the GAPDH and ACTB genes are the most widely used reference genes in human studies. However, GAPDH and ACTB are ranked as the least stable genes in the rhesus monkey. Genomic and environmental differences between humans and rhesus monkeys might result in the selection of different suitable HKGs in the six different tissues tested. Thus, different reference genes (RPL32 and RPL13A) should be used for the analysis of rhesus monkey tissue than those used for human tissue analysis

\section{Materials and methods \\ RNA samples}

Total RNA from rhesus macaque tissues (colon, liver, brain, lung, stomach, and kidney) and human total RNA (colon, liver, brain, lung, stomach, and kidney) were purchased from Clontech and Ambion, respectively. For the validation of our results for reference gene selection, corresponding unknown rhesus macaque tissues (colon, liver, brain, lung, stomach, and kidney) were provided by the National Primate Research Center (NPRC) of Korea. Total RNA was quantified using a NanoDrop ${ }^{\varpi}$ ND-1000 UV-Vis Spectrophotometer.

\section{Rhesus macaque and human tissue CDNA synthesis}

To eliminate DNA contamination from the total RNA samples, Turbo DNA-freeTM (Ambion) was used. A no-RT control was also amplified to confirm the absence of DNA contamination. M-MLV (Moloney-Murine Leukemia Virus) reverse transcriptase with an annealing temperature of $42^{\circ} \mathrm{C}$ was used for the reverse transcription reaction with RNase inhibiter (Promega).

\section{Candidate reference genes and primers for real-time quantitative $R T-P C R$}

Rhesus monkey reference genes were identified by comparison with human HKGs using the NCBI (National Center for Biotechnology Information) database and the UCSC (University of California Santa Cruz) genome browser (Table 1). Their sequences were used to design primers design using Primer3 software [25]. BLAST searches were performed to confirm the total gene specificity of the primer sequences, and the results showed the absence of multi-locus matching at the primer site. All primers except for RPL32 spanned at least one intron to avoid inaccuracy due to genomic DNA contamination in RNA samples. 


\section{Real-time quantitative $R T$-PCR}

SYBR green qRT-PCR was performed on a Rotor Gene 3000 (Corbett Research). In each run, 1 ul of cDNA was used as template for amplification per reaction. The sample was added to $19 \mathrm{ul}$ of reaction mixture containing $7 \mathrm{ul}$ $\mathrm{H}_{2} \mathrm{O}, 10$ ul QuantiTect ${ }^{\circledast}$ SYBR $^{\circledast}$ Green PCR Master Mix (Qiagen) and $1 \mathrm{ul}$ forward and reverse primers (Table 2). Real-time qRT-PCR amplification of the HKGs was carried out for 50 cycles of $94^{\circ} \mathrm{C}$ for $10 \mathrm{sec}, 58^{\circ} \mathrm{C}$ for $15 \mathrm{sec}$, and $72^{\circ} \mathrm{C}$ for $15 \mathrm{sec}$. The temperature range for the analysis of melting curves was $55^{\circ} \mathrm{C}$ to $99^{\circ} \mathrm{C}$ over $30 \mathrm{sec}$. Three independent experiments were performed.

\section{Characterization of analysis programs}

The geNorm [22] program provides a measure of gene expression stability $(M)$, which is the mean pair-wise variation between an individual gene and all other tested control genes. This method differs from model-based approaches by comparing genes based on the similarity of their expression profiles. Ct values were converted to scale expression quantities using the delta-Ct method and entered into geNorm, which then ranks the genes based on $M$ values where the gene with the most stable expression has the lowest value. NormFinder [23] focuses on finding the two genes with the least intra- and inter-group expression variation. A BestKeeper [24] index was created using the geometric mean of each candidate gene's $\mathrm{Ct}$ values. This index was then compared to each individual candidate housekeeping gene by pair-wise correlation analyses, with each combination assigned a value for the Pearson correlation coefficient (r) and the probability (p).

\section{Additional material}

\section{Additional file 1}

Standard curves for calculation of PCR efficiency and quantification according to reference genes in the rhesus monkey. Amplification of 10fold serial dilutions of the plasmid standard ranging from $10^{0}$ to $10^{5} \mathrm{copies}$ per reaction was carried out in duplicate.

Click here for file

[http://www.biomedcentral.com/content/supplementary/1471-

2199-9-78-S1.pdf]

\section{Additional file 2}

Standard curves for calculation of PCR efficiency and quantification according to reference genes in humans. Amplification of 10-fold serial dilutions of the plasmid standard ranging from $10^{0}$ to $10^{5}$ copies per reaction was carried out in duplicate.

Click here for file

[http://www.biomedcentral.com/content/supplementary/1471-

2199-9-78-S2.pdf]

\section{Additional file 3}

Efficiencies calculated from the slopes of dilution curves.

Click here for file

[http://www.biomedcentral.com/content/supplementary/1471-

2199-9-78-S3.xls]

\section{Additional file 4}

Melting curve analysis. Melting curve analysis of 8 different primer sets for reference genes in the rhesus monkey.

Click here for file

[http://www.biomedcentral.com/content/supplementary/1471-

2199-9-78-S4.pdf]

\section{Additional file 5}

Melting curve analysis. Melting curve analysis of 8 different primer sets for reference genes in humans.

Click here for file

[http://www.biomedcentral.com/content/supplementary/1471-

2199-9-78-S5.pdf]

\section{Additional file 6}

Selection of the most suitable reference genes for normalization using geNorm analysis using the unknown rhesus monkey samples for validation.

Click here for file

[http://www.biomedcentral.com/content/supplementary/14712199-9-78-S6.pdf]

\section{Additional file 7}

Candidate reference genes for normalization and the best combination of two genes listed according to their expression stability calculated by the

NormFinder program using the unknown rhesus monkey samples for validation.

Click here for file

[http://www.biomedcentral.com/content/supplementary/1471-

2199-9-78-S7.xls]

\section{Additional file 8}

Results from Bestkeeper descriptive statistical analysis using the unknown rhesus monkey samples for validation.

Click here for file

[http://www.biomedcentral.com/content/supplementary/1471-

2199-9-78-S8.xls]

\section{Acknowledgements}

This work was supported by the Korea Science and Engineering Foundation (KOSEF) grant funded by the Korea government (MOST) (No. ROI-2007000-20035-0).

\section{References}

I. Rhesus Macaque Genome Sequencing and Analysis Consortium: Evolutionary and Biomedical Insights from the Rhesus Macaque Genome. Science 2007, 316:222.

2. Vandesompele J, De Preter K, Pattyn F, Poppe B, Van Roy N, De Paepe A, Speleman F: Accurate normalization of real-time quantitative RT-PCR data by geometric averaging of multiple internal control genes. Genome Biol 2002, 3:34.

3. Nolan T, Hands RE, Bustin SA: Quantification of mRNA using real-time RT-PCR. Nat Protoc 2006, I(3): 1559-82.

4. Radonic A, Thulke S, Mackay IM, Landt O, Siegert W, Nitsche A: Guideline to reference gene selection for quantitative realtime PCR. Biochem Biophys Res Commun 2004, 3 I 3:856-862.

5. Sellars MJ, Vuocolo T, Leeton LA, Coman GJ, Degnan BM, Preston NP: Real-time RT-PCR quantification of Kuruma shrimp transcripts: a comparison of relative and absolute quantification procedures. J Biotechnol 2007, I 29(3):391-9. 
6. Schmittgen TD, Zakrajsek BA: Effect of experimental treatment on housekeeping gene expression: validation by real-time, quantitative RT-PCR. J Biochem Biophys Methods 2000, 46:69-8I.

7. Mogal A, Abdulkadir SA: Effects of Histone Deacetylase Inhibitor (HDACi); Trichostatin-A (TSA) on the expression of housekeeping genes. Mol Cell Probe 2006, 20:8I-86.

8. Li YP, Bang DD, Handberg KJ, Jorgensen PH, Zhang MF: Evaluation of the suitability of six host genes as internal control in realtime RT-PCR assays in chicken embryo cell cultures infected with infectious bursal disease virus. Vet Microbiol 2005, I I 0(34): $155-165$.

9. Ohl F, Jung M, Radoniæ A, Sachs M, Loening SA, Jung K: Identification and Validation of Suitable Endogenous Reference Genes for Gene Expression Studies of Human Bladder Cancer. Urology 2006, 175:1915-1920.

10. Fischer M, Skowron M, Berthold F: Reliable transcript quantification by real-time reverse transcriptase-polymerase chain reaction in primary neuroblastoma using normalization to averaged expression levels of the control genes HPRTI and SDHA. J Mol Diagn 2005, 7(I):89-96.

II. Goidin D, Mamessier A, Staquet MJ, Schmitt D, Berthier-Vergnes O: Ribosomal I8S RNA prevails over glyceraldehyde-3-phosphate dehydrogenase and beta-actin genes as internal standard for quantitative comparison of mRNA levels in invasive and noninvasive human melanoma cell subpopulations. Anal Biochem 200I, 295(I): I7-2I.

12. Goossens K, Van Poucke M, Van Soom A, Vandesompele J, Van Zeveren A, Peelman LI): Selection of reference genes for quantitative real-time $P C R$ in bovine preimplantation embryos. $B M C$ Dev Biol 2005, 5:2.

13. Kuijk EW, du Puy L, van Tol HT, Haagsman HP, Colenbrander B, Roelen BA: Validation of reference genes for quantitative RTPCR studies in porcine oocytes and preimplantation embryos. BMC Dev Biol 2007, 7:58.

14. Tricarico C, Pinzani P, Bianchi S, Paglierani M, Distante V, Pazzagli M, Bustin SA, Orlando C: Quantitative real-time reverse transcription polymerase chain reaction: normalization to rRNA or single housekeeping genes is inappropriate for human tissue biopsies. Anal Biochem 2002, 309:293-300.

15. Zhang X, Ding L, Sandford AJ: Selection of reference genes for gene expression studies in human neutrophils by real-time PCR. BMC Mol Biol 2005, 6:4.

16. Overbergh L, Kyama CM, Valckx D, Debrock S, Mwenda JM, Mathieu C, Hooghe TD: Validation of real-time RT-PCR assays for mRNA quantification in baboons. Cytokine 2005, 3 I (6):454-458.

17. Nygard $A B$, Jorgensen $C B$, Cirera $S$, Fredholm M: Selection of reference genes for gene expression studies in pig tissues using SYBR green qPCR. BMC Mol Biol 2007, 8:67.

18. Brinkhof B, Spee B, Rothuizen J, Penning LC: Development and evaluation of canine reference genes for accurate quantification of gene expression. Anal Biochem 2006, 356:36-43.

19. Spinsanti G, Panti C, Lazzeri E, Marsili L, Casini S, Frati F, Fossi CM: Selection of reference genes for quantitative RT-PCR studies in striped dolphin (Stenella coeruleoalba) skin biopsies. BMC Mol Biol 2006, 7:32.

20. Olsvik PA, Lie KK, Jordal AE, Nilsen TO, Hordvik I: Evaluation of potential reference genes in real-time RT-PCR studies of Atlantic salmon. BMC Mol Biol 2005, 6:2I.

21. Brunner AM, Yakovlev IA, Strauss SH: Validating internal controls for quantitative plant gene expression studies. $B M C$ PlantBiol 2004, 4: 14.

22. geNorm Software [http://medgen.ugent.be/ jvdesomp/genorm/]

23. NormFinder Software [http://www.mdl.dk/publication snormfinder.htm]

24. BestKeeperSoftware [http://www.gene-quantification.de/best keeper.html]

25. Rozen S, Skaletsky HJ: Primer3 on the WWW for general users and for biologist programmers. Bioinformatics Methods and Protocols: Methods in Molecular Biology 2000:365-386 [http:// frodo.wi.mit.edu/]. Totowa: Humana Press

26. Silver N, Best S, Jiang J, Thein SL: Selection of housekeeping genes for gene expression studies in human reticulocytes using real-time PCR. BMC Mol Biol 2006, 7:33.

27. Falk O, Monika J, Chuanliang $X$, Carsten S, Anja R, Mick B, Andreas $\mathrm{N}$, Glen K, Stefan AL, Aleksandar R, Klaus J: Gene expression stud- ies in prostate cancer tissue: which reference gene should be selected for normalization? J Mol Med 2005, 83:1014-1024.

28. Erkens T, Van Poucke M, Vandesompele J, Goossens K, Van Zeveren A, Peelman LI: Development of a new set of reference genes for normalization of real-time RT-PCR data of porcine backfat and longissimus dorsi muscle, and evaluation with PPARGCIA. BMC Biotechnol 2006, 6:4I.

29. Pfaffl MW, Tichopad A, Prgomet C, Neuvians TP: Determination of stable housekeeping genes, differentially regulated target genes and sample integrity: bestkeeper-excel-based tool using pair-wise correlations. Biotechnol Lett 2004, 26:509-5 I 5 .
Publish with BioMed Central and every scientist can read your work free of charge

"BioMed Central will be the most significant development for disseminating the results of biomedical research in our lifetime. "

Sir Paul Nurse, Cancer Research UK

Your research papers will be:

- available free of charge to the entire biomedical community

- peer reviewed and published immediately upon acceptance

- cited in PubMed and archived on PubMed Central

- yours - you keep the copyright 